\title{
Viewpoint \\ Dialysis dose in acute kidney injury: no time for therapeutic nihilism - a critical appraisal of the Acute Renal Failure Trial Network study
}

\author{
Claudio Ronco ${ }^{1,2}$, Dinna Cruz ${ }^{1,2}$, Helen Oudemans van Straaten ${ }^{3}$, Patrick Honore ${ }^{4}$, \\ Andrew House ${ }^{5}$, Du Bin 6 and Noel Gibney ${ }^{7}$ \\ ${ }^{1}$ Nephrology Department, St Bortolo Hospital, Viale Rodolfi 37, 36100 Vicenza, Italy \\ 2International Renal Research Institute Vicenza (IRRIV), Viale Rodolfi 37, 36100 Vicenza, Italy \\ ${ }^{3}$ Department of Intensive Care, Onze Lieve Vrouwe Gasthuis, Oosterpark 9, 1091 AC Amsterdam, the Netherlands \\ ${ }^{4}$ Intensive Care Unit, St-Pierre Para-Universitary Hospital, 9 Avenue Reine Fabiola, 1340 Ottignies-Louvain-la-Neuve, Belgium \\ ${ }^{5}$ London Health Sciences Centre, 339 Windermere Road, London, Ontario, Canada N6A 5A5 \\ ${ }^{6}$ Medical Intensive Care Unit, Peking Union Medical College Hospital, Shuaifuyuan 1, Wangfujing, Beijing, 100730, China \\ ${ }^{7}$ Division of Critical Care Medicine, University of Alberta, 3C1.12 Walter C. Mackenzie Centre, 8440-112 Street, Edmonton, Alberta T6G 2B7, Canada
}

Corresponding author: Claudio Ronco, Cronco@goldnet.it

Published: 16 October 2008

This article is online at http://ccforum.com/content/12/5/308

(C) 2008 BioMed Central Ltd
Critical Care 2008, 12:308 (doi:10.1186/cc7016)

\begin{abstract}
The optimal dialysis dose for acute kidney injury is a matter of great controversy. Clinical trials, predominantly single-center studies, have shown conflicting results. The Acute Renal Failure Trial Network (ATN) Study was designed to compare clinical outcomes between patients allocated to an intensive dose versus a less-intensive dose of renal replacement therapy. Recently, the results of this large randomized controlled multicenter study were published. The present article will discuss certain aspects of this trial: the overall design, the baseline patient characteristics, and comparison of the results with earlier studies. Finally, the article will address the implications of the ATN Study results for clinical practice.
\end{abstract}

\section{Introduction}

Since the original formulation of the dose concept for renal replacement therapy more than 30 years ago [1] and the establishment of a link between dose and clinical outcome $[2,3]$, individualized patient dosing based on urea clearance is now routine in end-stage renal disease (ESRD) patients. More recently, similar principles have been applied to critically ill patients with acute kidney injury (AKI) treated both with continuous renal replacement therapy (CRRT) and with intermittent modalities.

Using the normalized effluent rate as a dose surrogate in postdilution continuous venovenous hemofiltration, Ronco and colleagues reported higher survival in patients receiving a dose of 35 or $45 \mathrm{ml} /$ hour $/ \mathrm{kg}$ than in patients receiving a dose of $20 \mathrm{ml} /$ hour $/ \mathrm{kg}$ [4]. A second randomized controlled trial (RCT) demonstrated higher survival and renal recovery rate in AKI patients receiving an average of six intermittent hemodialysis (IHD) treatments per week compared with patients treated an average three times per week [5]. Since these two initial studies, three additional dose/outcome RCTs in CRRT [6-8] and one RCT in intermittent therapy [9] have been published, producing mixed results.

When the Acute Renal Failure Trial Network (ATN) Study was designed, only two of the CRRT dose/outcome studies $[4,6]$ and one of the intermittent therapy studies [5] had been published. The preponderance of evidence at that time suggested a clinical benefit for more aggressive dialysis dosing in AKI. A major limitation of the Ronco and colleagues [4] and Schiffl and colleagues [5] studies, however, along with the subsequent dose/outcome studies, was their predominant single-center design. Accordingly, the ATN investigators reasoned that a multicenter trial was required to clarify the effect of dialysis dose on AKI patient outcome. The investigators therefore embarked on a multicenter trial involving academic and Veterans Affairs hospitals in the United States, conducted over the time period of 2003 to 2007 . The study was published by the New England Journal of Medicine, in electronic form on 20 May 2008 and in print form on 3 July 2008 [10].

$\mathrm{AKI}=$ acute kidney injury; APACHE $=$ Acute Pathophysiology and Chronic Health Evaluation; ATN = Acute Renal Failure Trial Network; CRRT = continuous renal replacement therapy; cSOFA = cardiovascular component of Sepsis-related Organ Failure Assessment; CVVHDF = continuous venovenous hemodiafiltration; ESRD = end-stage renal disease; IHD = intermittent hemodialysis; RCT = randomized controlled trial; SLED = sustained low efficiency dialysis. 
The ATN Study failed to show a beneficial effect for higher dose of renal replacement therapy on the survival of AKI patients. This is an important finding and the investigators should be congratulated for their efforts. Nevertheless, because of the landmark nature of the trial, a critical analysis is certainly in order.

The purpose of the present editorial is several-fold. First, we shall discuss the overall design of the ATN Study, with emphasis on the fact that this trial evaluated the intensity of renal support and was not a comparison of different dialysis modalities. Second, we shall address the baseline patient characteristics, especially with regard to illness severity and the manner in which this influenced dialysis modality allocation. Third, we shall highlight the major results, including a comparison with the results of prior studies, and provide potential explanations for the different results. Finally, we discuss the implications of the ATN results for clinical practice.

\section{Design of the Acute Renal Failure Trial Network Study}

As opposed to previous dose/outcome RCTs, which were either single-center trials or dual-center trials, the ATN Study involved 27 American sites at which 1,124 patients were randomized to a relatively high dialysis dose (intensive dose group) or to a conventional dialysis dose (less-intensive dose group). Once this randomization based on dose occurred, the actual dialysis treatment modality was determined by illness severity, as estimated by the cardiovascular component of the Sepsis-related Organ Failure Assessment (cSOFA) [11]. IHD was prescribed for patients with relative hemodynamic stability at baseline (cSOFA score $=0,1$, or 2), while hemodynamically unstable patients (cSOFA score $=3$ or 4 ) were treated with either CRRT or sustained low efficiency dialysis (SLED). The initial modality assignment was not fixed, however, as patients could transition between IHD and CRRT/ SLED depending on their cSOFA score, although these transitions occurred strictly within the assigned dose group.

An intensive dose was defined as $35 \mathrm{ml} / \mathrm{kg} /$ hour (prescribed) in CRRT and as a delivered urea $K t / V$ value of 1.2 in each of six treatments per week in IHD and SLED. A less-intensive dose was defined as $20 \mathrm{mg} / \mathrm{kg} / \mathrm{hour}$ (prescribed) in CRRT and as a delivered urea $K \mathrm{t} / V$ value of 1.2 in each of three treatments per week for IHD and SLED. The primary endpoint for the study was all-cause mortality at 60 days, while recovery of renal function was an important secondary endpoint.

A detailed discussion of the urea $K t / V$ parameter is beyond the scope of the present paper, but this is the clinical standard for estimating chronic hemodialysis dose in ESRD patients [12]. The $K t / V$ parameter is essentially a dimensionless urea clearance that is normalized to patient body size (or, more specifically, to the urea distribution volume). An important point to note is that, although the relationship between the $K t / V$ value and patient survival has been explored exhaustively in ESRD, no such assessment in AKI patients had been performed prior to the ATN Study. Moreover, no clinical validation of any methodology for estimating the $\mathrm{Kt} / V$ parameter in AKI patients has ever been performed, and the approach used for this in the ATN Study was one developed for ESRD patients.

Failure to account for the qualitative and quantitative dose differences between IHD and CRRT precludes an appropriate interpretation of the ATN outcome data. Comparison between the CRRT and IHD doses is complex due to the nonsteady-state nature of the latter therapy. A physiologically logical approach to comparing the two modalities is the standardized urea $\mathrm{Kt} / V$ parameter, as described by Gotch [13]. This parameter provides a continuous equivalent estimate of weekly urea removal for disparate therapies. In the ATN Study, the weekly standardized urea $K \mathrm{t} / V$ value for intensive IHD can be estimated to be approximately 4.0 and that for less-intensive CRRT to be approximately 5.0. On a time-averaged basis, therefore, greater urea removal occurred in patients receiving less-intensive CRRT on a given day than in those receiving intensive IHD. This uncertain separation of the dose during periods of unknown duration makes failure to observe a treatment effect unsurprising in the study.

As indicated clearly above (and contrary to some misconceptions about the study), the ATN Study was not a comparison of different dialysis modalities. Instead, the ATN Study design was consistent with American clinical practice, in that a continuum of care approach for AKI (that is, matching the appropriate therapy to the patient's clinical status at different time points) was utilized. In this regard, the design implies IHD was not considered an appropriate modality for hemodynamically unstable patients by the investigators, the design of prior trials notwithstanding [14]. Of note, the relatively high rate of severe hypotensive events in patients treated with IHD (see below), despite being judged clinically 'hemodynamically stable', both supports the ATN approach to modality assignment and suggests that, from a hemodynamic point of view, a greater number of patients may have benefited from more liberal use of CRRT than that chosen for the study. On the other hand, the intent-to-treat trial design complicates analysis, as discussed subsequently.

Finally, an important inclusion criterion that merits mention relates to pre-existing chronic kidney disease. Male patients with a baseline serum creatinine $>2.0 \mathrm{mg} / \mathrm{dl}$ and female patients with a baseline serum creatinine $>1.5 \mathrm{mg} / \mathrm{dl}$ were excluded from the trial. The relatively low mean premorbid serum creatinine $(1.1 \mathrm{mg} / \mathrm{dl}$ in both groups) reflects this exclusion of patients with moderate to severe chronic kidney disease. This exclusion is an important consideration due to the high risk of progression to ESRD after an episode of AKI in the patient group with moderate to severe chronic kidney disease, especially in light of the relatively high rate of 
nonrenal recovery even after exclusion of these high-risk patients (see below).

\section{Patient baseline characteristics and dialysis modality allocation in the Acute Renal Failure Trial Network Study}

The intensive and less-intensive dose groups were very well matched overall with regard to demographic and clinical characteristics at the time of study enrollment. A point of emphasis, however, is that the baseline characteristics along with the patient outcomes within each dose group are really composite (blended) data provided by relatively stable patients (treated initially with IHD) and by very critically ill patients (treated initially with CRRT or SLED). Some notable features of the enrolled populations include the following.

The leading causes of $\mathrm{AKI}$ - as is usual - were ischemia, sepsis and multifactorial causes, with nephrotoxins being much less common.

Secondly, because the mean body weight before acute illness was $84 \mathrm{~kg}$, a reasonable assumption is that the actual body weight used for dosing (at least at the beginning of dialysis therapy) was minimally $90 \mathrm{~kg}$ - a figure consistent with several recent reports from the critically ill AKI patient population. Although specific data are not provided in the paper, a reasonable assumption is that weight gain might have been substantially greater in the patients initially treated with CRRT versus those treated with IHD due to their greater illness severity and more severe organ failure.

Thirdly, the average length of stay both in the hospital (approximately 10 to 11 days) and in the intensive care unit (6 to 7 days) prior to renal replacement therapy initiation was extremely long. Although the average duration of stay in the intensive care unit prior to CRRT initiation was even longer (8 days) in the recent CRRT dose/outcome study performed by Tolwani and colleagues [8], this duration has been much shorter in other studies. For instance, in a recent RCT comparing CRRT and IHD, the stay duration was only 2 to 3 days [14]. The timeliness of initiation of renal support in the ATN Study therefore needs to be considered when interpreting its findings. It should be noted that the protocol's allowance for one IHD treatment or up to 24 hours of CRRT prior to enrollment may have contributed to this relatively late start. Indeed, $65 \%$ of enrolled patients had received one renal replacement therapy session before study inclusion at a time when illness severity undoubtedly was at its peak in a substantial percentage. Moreover, this is a potentially important dose confounder, as there was no knowledge of the dose provided to these patients on the first day, when it probably mattered most.

Fourthly, the mean Acute Pathophysiology and Chronic Health Evaluation (APACHE) II score of approximately 26 suggests the CRRT/SLED patient group was quite critically ill. Based on the reported standard deviation and this mean value, the average APACHE II score in the CRRT/SLED subgroup was probably at least 28 . By comparison, the mean APACHE II scores were approximately 23, 25, and 26, respectively, in the studies of Ronco and colleagues [4], Saudan and colleagues [7], and Tolwani and colleagues [8]. A relatively high plasma creatinine, the result of both a predominantly male patient population and late renal replacement therapy initiation, may have contributed to the higher APACHE II score.

Finally, additional evidence supporting the overall high level of illness severity in the study is the high percentage (78\%) of patients who were oliguric.

In accordance with the baseline cSOFA score, CRRT or SLED was the initial modality in $55 \%$ of patients and IHD was the initial modality in $45 \%$ of patients. Among the most critically ill patients with hemodynamic instability at baseline (cSOFA score $=3$ or 4), however, CRRT was the clearly dominant modality. Specifically, in this group, CRRT represented $>95 \%$ of treatments while SLED was utilized in $<5 \%$ of treatments. This low utilization of SLED in hemodynamically unstable patients occurred despite the investigators' ability to prescribe either SLED or CRRT in the study. These findings corroborate large observational trials [15-17] that demonstrate CRRT is the standard of care for hemodynamically unstable patients with AKI. Moreover, these results suggest clinicians do not yet believe SLED is clinically equivalent to CRRT for the most critically ill patients, and the results also suggest that the probable use of SLED in this patient population has been overestimated, based on information appearing in the literature and provided at congresses.

\section{Characteristics of renal replacement therapies in the Acute Renal Failure Trial Network Study}

A total of 11,602 renal replacement treatments were provided in the study. As noted above, the number of treatments provided by SLED was very small (approximately $2.5 \%$ of all treatments). This section therefore includes the treatment characteristics for IHD and CRRT only.

\section{Intermittent hemodialysis}

A delivered $K t / V$ value of 1.2 was targeted, so the prescribed $K \mathrm{t} / \mathrm{V}$ level was in the 1.3 to 1.4 range per treatment to account for shortfalls in the delivery of the prescribed IHD dose. Of note, the mean delivered $K \mathrm{t} / \mathrm{V}$ value of 1.3 per treatment (after the first treatment) was actually higher than the targeted value. This feat is almost never accomplished in the real world, and previous studies have indicated that the delivered dose may fall short of the prescribed dose by $30 \%$ or more when provided in the intensive care unit for $\mathrm{AKI}$ $[18,19]$. This level of dose delivery required a mean treatment duration of 4 hours, a mean blood flow of $360 \mathrm{ml} /$ minute and a mean dialysate flow of $720 \mathrm{ml} /$ minute, all of which clearly 
represent a best-case scenario for IHD in this setting. Overall, it may be very difficult to apply these results in actual clinical practice, especially on a repeated basis.

The surprisingly low net ultrafiltration volumes achieved in the IHD patients are worth mentioning. The paper reported the average number of IHD treatments per week and the average net ultrafiltration volume per treatment. Based on the assumption that the cumulative net ultrafiltration volume over the period of 1 week is the product of these parameters, the weekly ultrafiltration volume was only 9.4 I (1.7 I/treatment $x$ 5.4 treatments/week) and $6.3 \mathrm{I}$ ( $2.1 \mathrm{l} /$ treatment $\times 3.0$ treatments/ week) in the intensive and less-intensive dose groups, respectively. Although the residual urine output could have supplemented the net fluid output in some patients, these ultrafiltration volumes are much lower than those achieved routinely in critically ill patients treated with CRRT - whose net volume removal requirements are usually at least 2.5 to 3 l/day, or approximately $20 \mathrm{l} /$ week. (In fact, among patients treated with CRRT, the mean daily net ultrafiltration volume per day was approximately $2.7 \mathrm{I}$ in both dose arms of the ATN Study.)

The relatively low illness severity of the IHD group may mean that these patients simply had lower volume removal requirements than the more critically ill patients treated with CRRT, or that large net ultrafiltrate volumes had already been achieved in those patients initially treated with CRRT and then transitioned to IHD. The potentially inadequate nature of the less-intensive IHD regimen with respect to fluid removal, however, is highlighted by the relatively high ratio (1:9) of offschedule isolated ultrafiltration treatments to actual IHD treatments. In addition, a contributing factor may have been the inability to achieve the prescribed volume removal due to hypotension, as discussed below.

Previous studies have not consistently confirmed the theoretical CRRT advantage of better preservation of hemodynamic stability relative to IHD, and a recent RCT suggested that IHD and CRRT can be used interchangeably in critically ill AKI patients [14]. The ATN hemodynamic data strongly challenge this notion, however, and suggest the incidence of hypotension in IHD has been generally underestimated in the past, despite advances in hemodialysis technology. Severe hypotension - defined as an episode necessitating discontinuation of treatment - occurred in $1.5 \%$ to $2 \%$ (average, $1.7 \%$ ) of all IHD treatments, and this frequency was not dependent on the treatment dose. By comparison of Supplementary Tables 5 and 6 from the New England Journal of Medicine publication [10], only $0.7 \%$ of CRRT treatments were terminated due to hypotension. The frequency at which severe hypotension occurred in the IHD-treated patients was therefore 2.5-fold greater than the same frequency in patients treated with CRRT - even though the latter group was more critically ill, more hemodynamically unstable, and required much higher net ultrafiltration volumes for fluid balance.
These hemodynamic data are particularly important in light of the continuing belief by many experts that any episode of hypotension in an AKI patient, even if very transient, can cause further kidney injury and possibly reduce the likelihood of renal recovery.

\section{Continuous renal replacement therapy}

More than $95 \%$ of all treatments provided to hemodynamically unstable patients consisted of continuous therapy in the study. It is important to note that, because the CRRT doses of $35 \mathrm{ml} / \mathrm{kg} / \mathrm{hour}$ and $20 \mathrm{ml} / \mathrm{kg} /$ hour were delivered with predilution continuous venovenous hemodiafiltration (CVVHDF), the effective delivered dose was less when the effect of dilution on actual solute clearance is considered [20,21] (see below). The average daily duration of therapy was approximately 21 hours in both groups, allowing for a high percentage of the prescribed dose to be delivered. Similar to the IHD dose delivery data, the relevance of these best-case results to standard clinical practice are unclear.

The average reported effluent volumes per day in the intensive and less-intensive dose groups were approximately 501 and $30 \mathrm{I}$, respectively, with an approximately equal contribution from dialysate (diffusion) and replacement (convection). These figures, however, are difficult to reconcile with the additional data provided. For the intensive dose group, the average hourly effluent rate, which is determined by summing the mean values of the individual components (dialysate rate, replacement rate, and net ultrafiltration rate), was $2,930 \mathrm{ml} /$ hour. When this value is multiplied by the mean daily duration of treatment for this group (20.9 hours), a mean daily effluent volume of $61.5 \mathrm{I}$ is determined. The reported mean daily effluent volume (50 I) is therefore approximately $20 \%$ less than the same parameter estimated in the above manner $(61.5 \mathrm{l})$. When the same calculations are performed for the less-intensive group, the reported mean daily effluent volume $(30 \mathrm{l})$ is also approximately $20 \%$ less than the calculated value (37.4 I).

Additional evidence raising questions about which CRRT dose was actually provided in the study can be derived from body-weight estimations. As suggested previously, one of the problems with the study is that the actual body weights used for CRRT dosing were not reported. Nevertheless, this average dosing weight can be estimated by dividing the reported mean hourly effluent rate $(2,930 \mathrm{ml}$ in the intensive dose group) by the mean delivered dose for this group $(35.8 \mathrm{ml} / \mathrm{hour} / \mathrm{kg})$. When this approach is followed, the calculated value for the mean body weight is $81.8 \mathrm{~kg}$. A similar calculation performed for the less-intensive dose group yields a mean body weight of $80.9 \mathrm{~kg}$. These calculated dosing body weights, however, are implausibly less than the reported mean premorbid weight (weight before acute illness) of $84.1 \mathrm{~kg}$. Based on an assumed $8 \%$ to $10 \%$ bodyweight increase in fluid volume from this premorbid weight, the actual body weight used for dosing CRRT may well have been 91 to $92 \mathrm{~kg}$, at least at therapy initiation. 


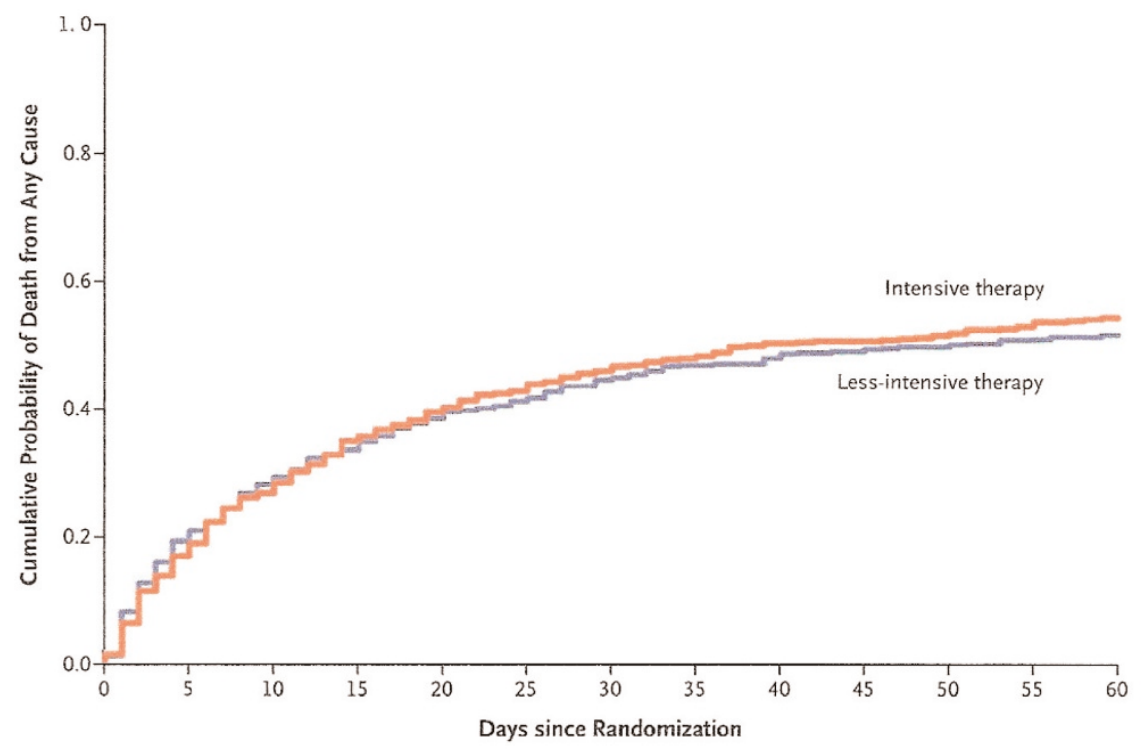

Effect of renal replacement dose on survival of acute kidney injury patients. Acute Renal Failure Trial Network Study. Reprinted with permission from [10].

In addition to these considerations that call into question which CRRT dose was actually delivered, the issue of effective dose reduction due to predilution must be addressed $[20,21]$. The combination of blood flow rates and replacement fluid flow rates used in the study suggest an effective dose reduction of approximately $15 \%$ in the intensive dose group and of approximately $9 \%$ in the less-intensive group. Furthermore, although the dose delivery was relatively high, still only $89 \%$ and $95 \%$ of the prescribed dose were delivered to the intensive and less-intensive dose groups, respectively. Therefore, even without consideration of above questions relating to the accuracy of the mean doses of 35.3 and $22 \mathrm{ml} / \mathrm{kg} /$ hour reported in the article for the intensive and less-intensive dose groups, respectively, the effective doses were at best only 27 and $19 \mathrm{ml} / \mathrm{kg} / \mathrm{hour}$, respectively.

Finally, it should be emphasized that use of the term dose in the study is limited to the clearance of a small solute (urea) in a specific CRRT modality (predilution CVVHDF). In this regard, it is instructive to compare the manner in which CRRT was delivered in the ATN Study with the manner in which it was delivered in the two single-center RCTs that reported a positive correlation between dose and survival. In one of these latter trials, since the CRRT modality used was postdilution continuous venovenous hemofiltration, effective dose decreases due to dilution effects were not relevant [4]. Furthermore, the percentage delivery of the prescribed dose was higher because increases in flow rates were prescribed to compensate for therapy down-time. Lastly, as a purely convective therapy, continuous venovenous hemofiltration provides a much broader solute removal spectrum from a molecular weight perspective than CVVHDF at similar effluent rates, and many $\mathrm{AKI}$ experts believe this contributed to the survival benefit observed with higher effluent rates in the study of Ronco and colleagues. In the second positive CRRT dose/outcome study [7], even though the modality associated with better survival was also the predilution CVVHDF, the dose was higher $(42 \mathrm{ml} / \mathrm{kg} /$ hour $)$ and a greater proportion of the dose was provided by convection rather than by diffusion, relative to the ATN Study.

\section{Major findings of the Acute Renal Failure Trial Network Study}

As mentioned previously, all-cause mortality was not different between the intensive dose group (53.6\%) and the lessintensive dose group (51.5\%) at 60 days (Figure 1$)$. It is critically important, however, to understand that the two curves shown in the figure are composite in nature because they incorporate data from patients initially treated with IHD (low cSOFA score) and those initially treated with CRRT or SLED (high cSOFA score). In this regard, it is interesting to note that intensive IHD (in relatively well patients) was associated with a $33 \%$ increase in death risk relative to less-intensive IHD. Conversely, intensive CRRT/SLED was associated with a $7 \%$ decrease in death risk relative to less-intensive therapy. Furthermore, in the oliguric and septic subgroups, the dose likewise did not have a significant effect on mortality. Finally, none of the secondary endpoints including inhospital mortality, renal recovery, and outcomes related to intensive care unit and hospital lengths of stay - was impacted significantly by treatment dose.

With regard to renal recovery, the percentage of patients who were considered to have no renal recovery was approximately $75 \%$ in both dose groups. Although this seems to be an 
inordinately high percentage on first inspection, two qualifications are in order. First, this figure is based on all patients enrolled in the trial while previous observational studies have limited assessment of this outcome to patients surviving to hospital discharge. Second, this outcome was measured at 28 days while the previous observational studies focused on this outcome have primarily used hospital discharge as the assessment time point.

The particular methodology used in the ATN Study artificially increased the rate of nonrenal recovery, relative to the aforementioned observational studies. On the other hand, even after accounting for the above differences, the rate of renal recovery at hospital discharge was estimated to be substantially lower (approximately 50\%) in the ATN Study than what has been reported previously $(60 \%$ to $90 \%)[16,17]$. Furthermore, the exclusion of patients with moderate to severe chronic kidney disease, who are at particular risk for progression to ESRD after AKI, most probably mitigated this result, at least to a certain extent. The clinical and health economic implications of renal nonrecovery after AKI are being increasingly recognized [22,23].

Although the dose did not have a significant impact on renal recovery, it is not clear from the reported data whether the dialysis modality played a role. This is a relevant question because recent large observational trials suggest that the use of CRRT results in a higher rate of renal recovery after AKI than the use of IHD $[16,17]$. Moreover, this issue of renal recovery raises a larger question about overall trial design. In an intent-to-treat design, patient outcomes are attributed to the initial treatment assignment - and in most studies employing this design, the initial treatment assignment is the only one that is relevant. In the ATN trial design, however, treatment transfers (CRRT/SLED to IHD or vice versa) occurred in a significant percentage of patients.

With regard to renal recovery, a specific scenario that causes concern is a patient initially treated with CRRT over a period of several days with progressively improving hemodynamic stability. Subsequently, this patient is treated with IHD, during which time several episodes of hypotension occur. If this particular patient fails to recover renal function, a probable cause would be the hypotension occurring during IHD. Owing to the intent-to-treat design, however, the nonrecovery of renal function would be attributed to CRRT. In light of the high rate of nonrecovery of renal function and the high incidence of hypotension in patients treated with IHD in the ATN Study, this is a highly relevant scenario and one that needs to be clarified further in future analyses by the ATN investigators.

\section{Clinical implications of the Acute Renal Failure Trial Network Study}

Based on the ATN Study's design, which does not easily permit the assessment of the separate effects of IHD and
Figure 2

\section{Renal Replacement Therapy}

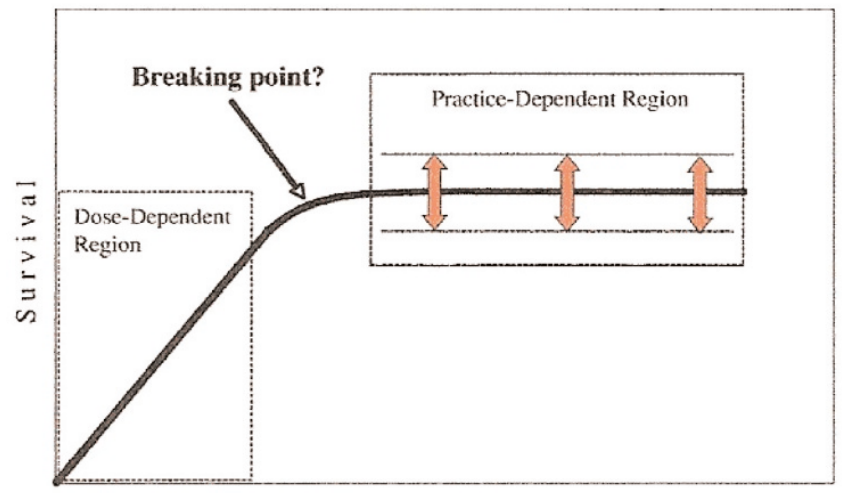

Dose of Dialysis (Urea or other adequacy markers)

Renal replacement dose/response relationship in acute kidney injury. Proposed by Ronco. Reprinted with permission from [24].

CRRT on patient outcome, it is not clear what impact the trial will have on clinical practice. Furthermore, at least with respect to CRRT, the effective treatment doses actually applied, especially to the intensive group, are unclear but are significantly different from previous studies showing a positive correlation between dose and survival.

From a more general perspective, as articulated recently [24], the manner in which data were interpreted from a previous American dose/outcome study performed in the late 1970s should serve as an argument against a rush to judgment. The National Cooperative Dialysis Study was the first study assessing the effect of the chronic hemodialysis dose on the outcome of ESRD patients [2]. Unfortunately, the results from this study were misinterpreted by the study investigators, who made ill-advised recommendations to the clinical community [25]. These recommendations most probably contributed to a systematic decrease in hemodialysis dose prescriptions in the United States and to an increased mortality over a prolonged period of time [26].

In general, as is the case with the dose of a drug, there is a dose/response effect for dialysis as it relates to an effect on survival. Even with the ATN data now available, however, the inflection point of the dose/survival curve at which further increases in treatment dose have no beneficial effect on survival has not been determined conclusively (Figure 2). Since this inflection point has not yet been established, we think it is wise to suggest caution in clinical practice [24]. We should encourage physicians not to make any significant changes to their current practice, and especially not to take decisions that may put patients at risk of underdialysis. We should not forget what we learnt from the experience with chronic dialysis, where dialysis dose was frankly over- 
estimated in the years following the National Cooperative Dialysis Study [25]: these years led to increased morbidity and mortality due to unscheduled underdialysis [26].

\section{Conclusion}

The ATN Study was a prodigious undertaking for which the investigators deserve enormous credit. Although the results clearly add to our understanding of the effect of dialysis dose on outcome in $\mathrm{AKI}$, a number of questions remain. These questions, many of which are addressed in the present editorial, preclude the development of specific recommendations in this area. Our recommendation, therefore, is that clinicians pursue a conservative approach that does not involve substantial changes in clinical practice. This is especially true for CRRT dosing in light of the ongoing dose/outcome RENAL trial in Australia and New Zealand, the results of which will probably consolidate the evidence and clarify the dose/outcome relationship.

\section{Competing interests}

The authors declare that they have no competing interests.

\section{References}

1. Teschan PE, Ginn HE, Bourne JR, Ward JW, Hamel B, Nunnally JC, Musso M, Vaughn WK: Quantitative indices of clinical uremia. Kidney Int 1979, 15:676-697.

2. Lowrie EG, Laird NM, Parker TF, Sargent JA: Effect of the hemodialysis prescription of patient morbidity: report from the National Cooperative Dialysis Study. N Engl J Med 1981, 305:1176-1181.

3. Hakim RM, Breyer J, Ismail N, Schulman G: Effects of dose of dialysis on morbidity and mortality. Am J Kidney Dis 1994, 23: 661-669.

4. Ronco C, Bellomo R, Hommel P, Brendolan A, Dan M, Piccinni P, LaGreca G: Effects of different doses in continuous venovenous hemofiltration on outcomes in acute renal failure: a prospective, randomized trial. Lancet 2000, 355:26-30.

5. Schiffl H, Lang SM, Fischer R: Daily hemodialysis and the outcome of acute renal failure. N Engl J Med 2002, 346:305310.

6. Bouman CS, Oudemans-Van Straaten HM, Tijssen JG, Zandstra DF, Kesecioglu J: Effects of early high-volume continuous veno-venous hemofiltration on survival and recovery of renal function in intensive care patients with acute renal failure: a prospective, randomized trial. Crit Care Med 2002, 30:22052211.

7. Saudan P, Niederberger M, De Seigneux S, Romand J, Pugin J, Perneger T, Martin PY: Adding a dialysis dose to continuous hemofiltration increases survival in patients with acute renal failure. Kidney Int 2006, 70:1312-1317.

8. Tolwani AJ, Campbell RC, Stofan BS, Lai KR, Oster RA, Wille KM: Standard versus high-dose CVVHDF for ICU-related acute renal failure. J Am Soc Nephrol 2008, 19:1233-1238.

9. Faulhaber-Walter R, Hafer C, Jahr N, Vahlbruch J, Hoy L, Haller H, Fliser D, Kielstein JT: The Hannover-Dialysis-Outcome (HANDOUT)-study: comparison of conventional versus intensified extended daily dialysis in treatment of patients with acute renal failure on the intensive-care unit [abstract]. $J \mathrm{Am}$ Soc Nephrol 2007, 18:802A-803A.

10. The VA/NIH Acute Renal Failure Trial Network: Intensity of renal support in critically ill patients with acute kidney injury. $N$ Engl J Med 2008, 359:7-20.

11. Vincent JL, Moreno R, Takala J, Willatts S, De Mendonça A, Bruining $\mathrm{H}$, Reinhart CK, Suter PM, Thijs LG: The SOFA (Sepsisrelated Organ Failure Assessment) score to describe organ dysfunction/failure. Intensive Care Med 1996, 22:707-710.

12. Clark WR, Rocco MV, Collins AJ: Quantification of hemodialysis: analysis of methods and relevance to clinical outcome. Blood Purif 1997, 15:92-111.
13. Gotch FA: The current place of urea kinetic modeling with respect to different dialysis modalities. Nephrol Dial Transplant 1998, 13(Suppl):10-14.

14. Vinsonneau C, Camus C, Combes A, Costa de Beauregard MA, Klouche K, Boulain T, Pallot JL, Chiche JD, Taupin P, Landais $P$, Dhainaut JF; Hemodiafe Study Group: Continuous venovenous haemodiafiltration versus intermittent hemodialysis for acute renal failure patients with multiple-organ dysfunction syndrome: a multicentre randomised trial. Lancet 2006, 368:379385.

15. Uchino S, Kellum JA, Bellomo R, Doig GS, Morimatsu H, Morgera S, Schetz M, Tan I, Bouman C, Macedo E, Gibney N, Tolwani A, Ronco C; Beginning and Ending Supportive Therapy for the Kidney (BEST Kidney) Investigators: Acute renal failure in critically ill patients: a multinational, multicenter study. JAMA 2005, 295:813-818.

16. Bell M, Granath F, Schon S, Ekbom A, Martling CR: Continuous renal replacement therapy is associated with less chronic renal failure than intermittent haemodialysis after acute renal failure. Intensive Care Med 2007, 33:773-780.

17. Uchino S, Bellomo R, Kellum JA, Morimatsu H, Morgera S, Schetz MR, Tan I, Bouman C, Macedo E, Gibney N, Tolwani A, Oudemans-Van Straaten HM, Ronco C; Beginning and Ending Supportive Therapy for the Kidney (B.E.S.T. Kidney) Investigators Writing Committee: Patient and kidney survival by dialysis modality in critically ill patients with acute kidney injury. Int $J$ Artif Organs 2007, 30:281-292.

18. Evanson JA, Himmelfarb J, Wingard R, Knights S, Shyr Y, Schulman G, Ikizler TA, Hakim RM: Measurement of the delivery of dialysis in acute renal failure. Kidney Int 1999, 55:1501-1508.

19. Clark WR, Ronco C: Factors influencing therapy delivery in acute dialysis. Contrib Nephrol 2001, 132:304-312.

20. Clark WR, Ronco C: Continuous renal replacement techniques. Contrib Nephrol 2004, 144:264-277.

21. Huang Z, Letteri JJ, Clark WR, Ronco C: Operational characteristics of continuous renal replacement therapy modalities used for critically ill patients with acute kidney injury. Int J Artif Organs 2008, 31:525-534.

22. Ronco C, Bagshaw SM, Gibney RT, Bellomo R: Outcome comparisons of intermittent and continuous therapies in acute kidney injury: what do they mean? Int J Artif Organs 2008, 31: 213-220.

23. Clark WR, Letteri JJ, Uchino S, Bellomo R, Ronco C: Recent clinical advances in the management of critically ill patients with acute renal failure. Blood Purif 2006, 24:487-498.

24. Ronco C: What if the ATN trial ...? Int J Artif Organs 2008, 31: 379-381.

25. Gotch FA, Sargent FA: A mechanistic analysis of the National Cooperative Dialysis Study (NCDS). Kidney Int 1985, 28:526534.

26. Acchiardo SR, Hatten KW, Ruvinsky MJ, Dyson B, Fuller J, Moore LW: Inadequate dialysis increases gross mortality rate. ASAIO J 1992, 38:M282-M285. 